\title{
DIFFERENTIABLE ACTIONS ON HOMOTOPY SEVEN SPHERES
}

\author{
BY \\ D. MONTGOMERY AND C. T. YANG(1)
}

To A. D. Wallace on his sixtieth birthday

1. Introduction. This paper studies differentiable actions of the circle group SO(2) on homotopy 7 -spheres. The class of free such actions is shown to form an infinite abelian group which is closely related to the group of differentiably knotted 3-spheres in the 6-sphere. We also consider actions, each having a 3-sphere as the fixed point set and acting freely otherwise. Some of our results are as follows. (The appendix shows that recent work of Haefliger gives additional results.)

THEOREM 1. The equivariant diffeomorphism classes of free differentiable actions of the circle group on homotopy seven spheres form an abelian group which contains an element of infinite order.

THEOREM 2. On any homotopy seven sphere, there are infinitely many differentiably distinct free differentiable actions of the circle group.

THEOREM 3. On any homotopy seven sphere, there are infinitely many differentiably distinct differentiable actions of the circle group, each having a three sphere as the fixed point set and acting freely otherwise.

Theorem 2 has been proved for the 11-sphere by a different approach [1]. Further examples are given by W. C. Hsiang, $A$ note on free differentiable actions of $S^{1}$ and $S^{3}$ on homotopy spheres, Ann. of Math. 83 (1966), 266-272. Whether or not there is a similar result for the 5-sphere is open.

In order to obtain the group mentioned in Theorem 1 we work with the quotient spaces and introduce in these spaces a sum operation which is somewhat analogous to, but is distinct from, the ordinary connected sum. Use will be made of the fact that each free differentiable action of the circle group $\mathrm{SO}(2)$ on a homotopy 7-sphere determines a simply connected differentiable 6-manifold with the cohomology properties of the complex projective 3-space, and conversely that such a manifold determines such an action.

After preliminary remarks, two standard actions of $\mathrm{SO}(2)$ on the 7 -sphere are given. In a sense each of these can be obtained from the other by surgery. It is this idea that suggests our sum operation.

Presented to the Society, November 13, 1965; received by the editors May 12, 1965.

(1) The second author is supported in part by the U. S. Army Research Office. 
Throughout the paper, differentiability is in the $C^{\infty}$-sense and homology and cohomology are in the sense of singular theory with coefficients in the ring $Z$ of integers. Also we let

$R^{n}=$ euclidean $n$-space,

$D^{n}=\left\{x \in R^{n}|| x \mid \leqq 1\right\}$, i.e., the unit closed $n$-disk in $R^{n}$,

$S^{n-1}=$ boundary of $D^{n}$, i.e., the unit $(n-1)$-sphere in $R^{n}$,

$E^{n}=D^{n}-S^{n-1}$, i.e., the unit open $n$-disk in $R^{n}$, where all are understood to be the differentiable manifolds having the standard differentiable structures. For terminology such as imbeddings, diffeomorphisms, diffeotopies, strong diffeotopies, we refer to [2]. For the sake of convenience, we let the circle group be denoted by $G$ and consist of complex numbers $g$ with $|g|=1$, and let $R^{4}$ be the quaternion field. Whenever $g \in G$ and $q \in R^{4}$, we let $g q$ be the product of $g$ and $q$. Then we have a differentiable action of $G$ on $R^{4}$ by a left multiplication which leaves 0 fixed acts freely otherwise. Under this action, we may set,

$$
D^{3}=D^{4} / G=\left\{G q \mid q \in D^{4}\right\}, \quad S^{2}=S^{3} / G .
$$

\section{Two standard actions of $G$ on $S^{7}$.}

First standard action of $G$ on $S^{7}$. Whenever $g \in G$ and $(u, v) \in R^{8}$, where $u$, $v \in R^{4}$, we let

$$
g \cdot(u, v)=(g u, g v) .
$$

Then we have a free differentiable action of $G$ on $S^{7}$ and the orbit space $\left\{G \cdot(u, v) \mid(u, v) \in S^{7}\right\}$ is the complex projective 3-space, abbreviated by $C P^{3}$, which has an integral cohomology ring

$$
H^{*}\left(C P^{3}\right)=Z[\alpha] /\left(\alpha^{4}\right),
$$

where $\alpha \in H^{2}\left(C P^{3}\right)$. The manifold $C P^{3}$ is understood to have the standard differentiable structure, i.e., the one given by the quotient structure.

Let

$$
h, h^{\prime}: S^{2} \times D^{4} \rightarrow C P^{3}
$$

be the imbeddings defined by $h(G u, v)=G \cdot(a u, a u v)$ and $h^{\prime}(G u, v)=G \cdot(a u v, a u)$, where $a=\left(1+|v|^{2}\right)^{-1 / 2}$. Then

$$
\begin{gathered}
h\left(S^{2} \times D^{4}\right) \cup h^{\prime}\left(S^{2} \times D^{4}\right)=C P^{3}, \\
h\left(S^{2} \times D^{4}\right) \cap h^{\prime}\left(S^{2} \times D^{4}\right)=h\left(S^{2} \times S^{3}\right)=h^{\prime}\left(S^{2} \times S^{3}\right)
\end{gathered}
$$

and

$$
f: S^{2} \times S^{3} \rightarrow S^{2} \times S^{3}
$$

defined by $f=h^{\prime-1} h$, is a diffeomorphism and is given by $f(G u, v)=\left(G u v, v^{-1}\right)$. Notice that $f^{-1}=f$. 
Let

$$
p: S^{2} \times S^{3} \rightarrow S^{2}, \quad p^{\prime}: S^{2} \times S^{3} \rightarrow S^{3}
$$

be projections and let

$$
q: S^{3} \rightarrow S^{2} \times S^{3}
$$

be defined by $q(v)=(G u, v)$, where $G u$ is a preassigned point of $S^{2}$. It is easily seen that

$$
\begin{gathered}
p f q: S^{3} \rightarrow S^{2} \text { represents a generator of } \pi_{3}\left(S^{2}\right) \\
\text { and } p^{\prime} f q: S^{3} \rightarrow S^{3} \text { is of degree }-1 .
\end{gathered}
$$

Second standard action of $G$ on $S^{7}$. Whenever $g \in G$ and $(u, v) \in R^{8}$, where $u, v \in R^{4}$, we let

$$
g \circ(u, v)=(g u, v) .
$$

Then again we have a differentiable action of $G$ on $S^{7}$. Under this action, $G$ leaves all points $\{0\} \times S^{3}$ fixed and acts freely otherwise. Moreover, the orbit space $\left\{G \circ(u, v) \mid(u, v) \in S^{7}\right\}$, which is assigned the differentiable structure such that the projection of $S^{7}$ into the orbit space is differentiable, is diffeomorphic to $S^{6}$ so that we may set it equal to $S^{6}$. Furthermore, $\{0\} \times S^{3}$ is the image of an imbedding of $S^{3}$ into $S^{6}$ and is differentiably unknotted in $S^{6}$ so that we may set it equal to $S^{3}$. Since there are two orbit types, it is more revealing for the action to have a pair $\left(S^{6}, S^{3}\right)$ rather than $S^{6}$ alone.

Let

$$
k: S^{2} \times D^{4} \rightarrow S^{6}, k^{\prime}: D^{3} \times S^{3} \rightarrow S^{6}
$$

be the imbeddings defined by $k(G u, v)=G \circ(a u, a v)$ and $k^{\prime}(G u, v)=G \circ(b u, b v)$ where $a=\left(1+|v|^{2}\right)^{-1 / 2}$ and $b=\left(1+|u|^{2}\right)^{-1 / 2}$. Then

$$
\begin{gathered}
k\left(S^{2} \times D^{4}\right) \cup k^{\prime}\left(D^{3} \times S^{3}\right)=S^{6}, \\
k\left(S^{2} \times D^{4}\right) \cap k^{\prime}\left(D^{3} \times S^{3}\right)=k\left(S^{2} \times S^{3}\right)=k^{\prime}\left(S^{2} \times S^{3}\right)
\end{gathered}
$$

and

$$
i: S^{2} \times S^{3} \rightarrow S^{2} \times S^{3}
$$

defined by $i=k^{\prime-1} k$, is the identity diffeomorphism.

3. Homotopy complex projective 3-spaces. By a homotopy complex projective 3-space, abbreviated by $\mathrm{HCP}^{3}$, we mean a closed differentiable 6-manifold which is simply connected and has the integral cohomology ring isomorphic to $\mathrm{H}^{*}\left(C P^{3}\right)$ (see (2)). Clearly $C P^{3}$ is an $\mathrm{HCP}^{3}$ and every $\mathrm{HCP}^{3}$ is of the same homotopy type as $C P^{3}$. Whenever $M$ is an $\mathrm{HCP}^{3},[M]$ denotes the diffeomorphism class of $M$. The set of all diffeomorphism classes is denoted by $\Pi$. 
By a $(6,3)$-sphere pair, we mean a pair $(P, Q)$ in which $P$ is a differentiable 6-manifold diffeomorphic to $S^{6}$ and $Q$ is the image of an imbedding of $S^{3}$ into $P$. Clearly the pair $\left(S^{6}, S^{3}\right)$ given in $\S 2$, is a $(6,3)$-sphere pair. Whenever $(P, Q)$ is a $(6,3)$-sphere pair, we denote by $[P, Q]$ the diffeomorphism class of $(P, Q)$. The set of all diffeomorphism classes of $(6,3)$-sphere pairs is denoted by $\Sigma$.

Denote by $\Sigma^{6,3}$ the set of $h$-cobordism classes of $(6,3)$-sphere pairs. In [3] $\Sigma^{6,3}$ is made an abelian group with a binary operation induced by a connected sum and it is shown that there is a homomorphism of $\Sigma^{6,3}$ onto $Z$. It is easily seen that $\Sigma$ can be made an abelian group in the same way and that there is a homomorphism of $\Sigma$ onto $\Sigma^{6,3}$ mapping $[P, Q]$ into the cobordism class of $(P, Q)$. Hence $\Sigma$ is an abelian group containing an element of infinite order. In order to use these results, we construct a map from $\Pi$ to $\Sigma$ suggested by the correspondence of $C P^{3}$ and $\left(S^{6}, S^{3}\right)$ as will be seen in $\S 4$. Since there is a group structure on $\Sigma$, it is natural to have a group structure on $\Pi$ also. Therefore we introduce a new kind of sum operation for $\mathrm{HCP}^{3}$ 's used to induce a group operation on $\Pi$.

We deal with only orientable differentiable manifolds, with or without boundary. For the sake of convenience, we let all of them be oriented. For $S^{n}$, we denote by $\alpha_{n}$ (respectively $\beta_{n}$ ) the generator of $H^{n}\left(S^{n}\right)$ (respectively $H_{n}\left(S^{n}\right)$ ) which represents the orientation on $S^{n}$. Therefore $\left\langle\alpha_{n}, \beta_{n}\right\rangle=1$. For an $M$ which is an $\mathrm{HCP}^{3}$, we denote by $\alpha_{M}$ the generator of $H^{2}(M)$ such that $\alpha_{M}^{3}$ represents the orientation on $M$, and by $\beta_{M}$ the generator of $H_{2}(M)$ such that $\left\langle\alpha_{M}, \beta_{M}\right\rangle=1$. For the sake of simplicity, we use $\alpha$ to mean $\alpha_{2}$ or $\alpha_{C P^{3}}$ and use $\beta$ to mean $\beta_{2}$ or $\beta_{C P^{3}}$.

4. Pasting process. Let $X$ and $Y$ be differentiable manifolds with boundary and let $\lambda$ be a diffeomorphism of the boundary $A$ of $X$ onto the boundary $B$ of $Y$. Then we can have a differentiable manifold

$$
X \cup_{\lambda} Y
$$

obtained from the disjoint sum $X+Y$ by identifying $x$ with $\lambda(x)$ for all $x \in A$, where the differentiable structure on $X \cup_{\lambda} Y$ is constructed by applying a standard smoothing procedure when the differentiable structures on $X$ and $Y$ are combined.

If $h, h^{\prime}: S^{2} \times D^{4} \rightarrow C P^{3}$ are the imbeddings of (3) and

$$
\lambda: h\left(S^{2} \times S^{3}\right) \rightarrow h^{\prime}\left(S^{2} \times S^{3}\right)
$$

is the identity diffeomorphism, then

$$
C P^{3}=h\left(S^{2} \times D^{4}\right) \cup_{\lambda} h^{\prime}\left(S^{2} \times D^{4}\right) .
$$

Therefore, if $f$ is the diffeomorphism of (4), then

$$
\left(S^{2} \times D^{4}\right) \cup_{f}\left(S^{2} \times D^{4}\right)
$$


is diffeomorphic to $C P^{3}$. Similarly, if $k: S^{2} \times D^{4} \rightarrow S^{6}$ and $k^{\prime}: D^{3} \times S \rightarrow S^{6}$ are the imbeddings of (8) and $\lambda: k\left(S^{2} \times S^{3}\right) \rightarrow k^{\prime}\left(S^{2} \times S^{3}\right)$ is the identity diffeomorphism, then

$$
S^{6}=k\left(S^{2} \times D^{4}\right) \cup_{\lambda} k^{\prime}\left(D^{3} \times S^{3}\right) .
$$

Therefore, if $i: S^{2} \times S^{3} \rightarrow S^{2} \times S^{3}$ is the identity diffeomorphism, then

$$
\left(S^{2} \times D^{4}\right) \cup\left(D^{3} \times S^{3}\right)
$$

is diffeomorphic to $S^{6}$ under a diffeomorphism mapping $\{0\} \times S^{3}$ onto $S^{3}$.

From (9) and (10), it is obvious that up to a diffeomorphism, $\left(S^{6}, S^{3}\right)$ can be obtained from $C P^{3}$ by a surgery in which $S^{2} \times D^{4}$ (one copy only!) is replaced by $D^{3} \times S^{3}$ and conversely that $C P^{3}$ can be recovered from $\left(S^{6}, S^{3}\right)$ by reversing the surgery. It is this surgery we shall use to construct a correspondence between $\mathrm{HCP}^{3}$ 's and $(6,3)$-sphere pairs.

Let $M$ and $N$ be connected oriented differentiable $n$-manifolds without boundary. Then the ordinary connected sum of $M$ and $N$ can be constructed as follows: Let $h: D^{n} \rightarrow M$ and $k: D^{n} \rightarrow N$ be orientation-preserving imbeddings,

$$
f: S^{n-1} \rightarrow S^{n-1}
$$

the reflection with respect to a hyperplane through the origin and

$$
\lambda: h\left(S^{n-1}\right) \rightarrow k\left(S^{n-1}\right)
$$

the diffeomorphism defined by $\lambda=k f h^{-1}$. Then the ordinary connected sum of $M$ and $N$ is defined to be

$$
M \# N=\left(M-h\left(E^{n}\right)\right) \cup_{\lambda}\left(N-k\left(E^{n}\right)\right)
$$

which, up to a diffeomorphism, is independent of $h$ and $k$ so that it is uniquely determined by $M$ and $N$. Since

$$
D^{n} \cup_{f} D^{n} \text { is diffeomorphic to } S^{n},
$$

it follows that $M \# S^{n}$ and $S^{n} \# M$ are diffeomorphic to $M$.

There is a similarity occurring in this paper concerning $\mathrm{HCP}^{3}$ 's. In fact, we shall show that if $M$ and $N$ are $\mathrm{HCP}^{3}$ 's, $h: S^{2} \times D^{4} \rightarrow M$ and $k: S^{2} \times D^{4} \rightarrow N$ are primary imbeddings (see next section), $f$ is the diffeomorphism (4) and $\lambda: h\left(S^{2} \times S^{3}\right) \rightarrow k\left(S^{2} \times S^{3}\right)$ is defined by $\lambda=k f h^{-1}$, then

$$
M * N=\left(M-h\left(S^{2} \times E^{4}\right)\right) \cup_{\lambda}\left(N-k\left(S^{2} \times E^{4}\right)\right)
$$

is an $\mathrm{HCP}^{3}$ which, up to a diffeomorphism, is uniquely determined by $M$ and $N$ (Lemma 9). The manifold $M * N$ is called the sum of $M$ and $N$. In place of (11), we have (9) instead. Therefore 


$$
M * C P^{3} \text { and } C P^{3} * M
$$

are diffeomorphic to $M$. This sum operation will be used to induce a group operation in the set $\Pi$ of all diffeomorphism classes of $\mathrm{HCP}^{3}$ 's such that the zero of the group is the diffeomorphism class of $C P^{3}$.

5. Primary imbeddings. Let $M$ be an $\mathrm{HCP}^{3}$. By a primary imbedding of $S^{2}$ into $M$, we mean an imbedding $j: S^{2} \rightarrow M$ such that $j_{*} \beta=\beta_{M}$. (For $\beta$ and $\beta_{M}$, see $\S 3$.) By a primary imbedding of $S^{2} \times D^{4}$ into $M$, we mean an orientation-preserving imbedding $h: S^{2} \times D^{4} \rightarrow M$ such that $h r: S^{2} \rightarrow M$ is a primary imbedding, where

$$
r: S^{2} \rightarrow S^{2} \times D^{4}
$$

is the imbedding defined by $r(x)=(x, 0)$.

Lemma 1. Let $M$ be an $H C P^{3}$ and let $U$ be a nonnull connected simply connected open subset of $M$ such that the homomorphism $\mathrm{H}_{2}(U) \rightarrow \mathrm{H}_{2}(M)$ induced by the inclusion map is an isomorphism onto. Then there is a primary imbedding $j: S^{2} \rightarrow M$ with $j\left(S^{2}\right) \subset U$.

Proof. Since $U$ is nonnull, connected and simply connected, it follows from the Hurewicz isomorphism theorem [4, p. 57] that $\pi_{2}(U)$ is isomorphic to $H_{2}(U)$ so that there is a map $j^{\prime}: S^{2} \rightarrow U$ such that $j_{*}^{\prime}: H_{2}\left(S^{2}\right) \rightarrow H_{2}(U)$ is an isomorphism onto. Since $2 \operatorname{dim} S^{2}<\operatorname{dim} U=\operatorname{dim} M, j^{\prime}$ can be approximated by an imbedding $j^{\prime \prime}: S^{2} \rightarrow U$ homotopic to $j^{\prime}$ (see, for example, [5]). Hence our assertion follows.

As a special case of Lemma 1, we have

Lemma 2. Whenever $M$ is an $H C P^{3}$, there is a primary imbedding $j: S^{2} \rightarrow M$.

Lemma 3. Whenever $M$ is an $H C P^{3}$ and $j: S^{2} \rightarrow M$ is a primary imbedding, there is a primary imbedding $h: S^{2} \times D^{4} \rightarrow M$ such that $j=h r$, where $r$ is the imbedding of (13).

Proof. Let there be a Riemannian metric on $M$. Since $S=j\left(S^{2}\right)$ is a submanifold of $M$, we have a normal bundle of $S$ in $M$, say

$$
\pi: n(M, S) \rightarrow S .
$$

If we are able to show that the normal bundle is a product bundle, then there is a bundle equivalence $k: S^{2} \times R^{4} \rightarrow n(M, S)$. Since there is a $\delta>0$ such that the exponential exp: $n(M, S) \rightarrow M$ maps $T=\{v \in n(M, S)|| v \mid \leqq \delta\}$ diffeomorphically onto a closed tubular neighborhood of $S$ in $M$, it follows that $h: S^{2} \times D^{4} \rightarrow M$ defined by $h(x, y)=\exp k(x, \delta y)$, is a desired imbedding.

It is known that $\pi: n(M, S) \rightarrow S$ can be decomposed into the Whitney sum of an $R^{3}$-bundle $\pi^{\prime}: n^{\prime}(M, S) \rightarrow S$ and a product $R^{1}$-bundle $\pi^{\prime \prime}: n^{\prime \prime}(M, S) \rightarrow S$, that means, for any $u \in S, \pi^{-1} u$ and $\pi^{\prime \prime-1} u$ are linear subspaces of $\pi^{-1} u$ and $\pi^{-1} u=\pi^{\prime-1} u \oplus \pi^{\prime \prime} u[6$, p. 135]. Therefore it is sufficient to show that 
$\pi^{\prime}: n^{\prime}(M, S) \rightarrow S$ is a product bundle. Let $T^{\prime}=T \cap n^{\prime}(M, S)$ and let $B$ be the boundary of $T^{\prime}$. Then $\pi^{\prime}: B \rightarrow S$ is a 2 -sphere bundle over $S$. Since $\pi^{\prime}: T^{\prime} \rightarrow S$ is a 3-disk bundle over a 2-sphere, it follows that $H^{4}\left(T^{\prime}\right)=0$.

If $\pi^{\prime}: n^{\prime}(M, S) \rightarrow S$ is not a product bundle, then neither is $\pi^{\prime}: B \rightarrow S$. Therefore there is a cross-section $c: S \rightarrow B$ such that $c(S)$ has a self intersection number 1 in $B\left[6\right.$, p. 137]. Since $j: S^{2} \rightarrow M$ is a primary imbedding, $\kappa^{*} \alpha_{M}$ is a generator of $H^{2}(S)$, where $\kappa$ is the inclusion map of $S$ into $M$. Therefore there is a generator $e$ of $H_{2}(S)$ such that $\left\langle\kappa^{*} \alpha_{M}, e\right\rangle=1$. Let $\xi=\exp \mid B$. Then $\kappa, \xi c: S \rightarrow M$ are homotopic so that

$$
1=\left\langle\kappa^{*} \alpha_{M}, e\right\rangle=\left\langle c^{*} \xi^{*} \alpha_{M}, e\right\rangle=\left\langle\xi^{*} \alpha_{M}, c_{*} e\right\rangle .
$$

Since $c(S)$ has a self intersection number 1 in $B$, it follows from Alexander-Pontrjagin duality on $B$ that $\xi^{*} \alpha_{M}^{2}=\left(\xi^{*} \alpha_{M}\right)^{2}$ is a generator of $H^{4}(B)$ so that $\xi^{*} \alpha_{M}^{2} \neq 0$. On the other hand, $\xi$ is the composition of the inclusion map $\xi^{\prime}: B \rightarrow T^{\prime}$ and $\xi^{\prime \prime}=\exp \mid T^{\prime}$ and $\xi^{\prime \prime *}$ is trivial (because $H^{4}\left(T^{\prime}\right)=0$ ). We infer that

$$
\xi^{*} \alpha_{M}^{2}=\xi^{* * \xi^{\prime \prime}} \alpha_{M}^{2}=0,
$$

contrary to the result just obtained. Hence the proof of Lemma 3 is completed.

LEMMA 4. Any two primary imbeddings of $\mathrm{S}^{2} \times D^{4}$ into an $\mathrm{HCP}^{3}$ are strongly diffeotopic.

Proof. Let $M$ be an $\mathrm{HCP}^{3}$ and let $h$ and $h^{\prime}$ be primary imbeddings of $S^{2} \times D^{4}$ into $M$. Then $h r$ and $h^{\prime} r$ are primary imbeddings of $S^{2}$ into $M$, where $r$ is the imbedding of (13). By the Hurewicz isomorphism theorem, $h r$ and $h^{\prime} r$ represent the same element of $\pi_{2}(M)$ so that they are homotopic. Therefore, by Haefliger's theorem [5], $h r$ and $h^{\prime} r$ are strongly diffeotopic, that means, there is a strong diffeotopy $H: M \times[0,1] \rightarrow M$ such that, if for each $t \in[0,1], H_{t}: M \rightarrow M$ is the diffeomorphism defined by $H_{t}(u)=H(u, t)$, then $H_{0}$ is the identity and $H_{1} h r=h^{\prime} r$. Now $H_{1} h, h^{\prime}: S^{2} \times D^{4} \rightarrow M$ are imbeddings such that the images of tubular neighborhoods of $h^{\prime} r\left(S^{2}\right)=h^{\prime}\left(S^{2} \times\{0\}\right)$ and for any $x \in S^{2}, H_{1} h(x, 0)=h^{\prime}(x, 0)$. Therefore there is, by [2, Theorem 5.2], a strong diffeotopy $H^{\prime}: M \times[0,1] \rightarrow M$ such that, if for each $t \in[0,1], H_{t}^{\prime}: M \rightarrow M$ is the diffeomorphism defined by $H_{t}^{\prime}(u)=H^{\prime}(u, t)$, then $H_{0}^{\prime}$ is the identity and there is a differentiable map $\phi: S^{2} \rightarrow \mathrm{SO}(4)$ with $H_{1}^{\prime} H_{1} h(x, y)=h^{\prime}(x, \phi(x) y)$ for all $(x, y) \in S^{2} \times D^{4}$. Since $\pi_{2}(\mathrm{SO}(4))=0, \phi$ is homotopic to the constant map which maps $S^{2}$ into the identity of $\mathrm{SO}(4)$. Therefore there is a strong diffeotopy $H^{\prime \prime}: M \times[0,1] \rightarrow M$ such that, if $H_{t}^{\prime \prime}: M \rightarrow M$ is the diffeomorphism defined by $H_{t}^{\prime \prime}(u)=H^{\prime \prime}(u, t)$, then $H_{0}^{\prime \prime}$ is the identity and $h^{\prime}(x, y)=H_{1}^{\prime \prime} h^{\prime}(x, \phi(x) y)$ for all $(x, y) \in S^{2} \times D^{4}$. Hence $H_{1}^{\prime \prime} H_{1}^{\prime} H_{1} h=h^{\prime}$ proving that $h$ and $h^{\prime}$ are strongly diffeotopic under $H^{\prime \prime} H^{\prime} H$.

Lemma 5. Whenever $M$ is an $H C P^{3}$ and $h: S^{2} \times D^{4} \rightarrow M$ is a primary imbedding, there is a primary imbedding $h^{\prime}: S^{2} \times D^{4} \rightarrow M$ such that 


$$
\begin{aligned}
& h\left(S^{2} \times D^{4}\right) \cup h^{\prime}\left(S^{2} \times D^{4}\right)=M, \\
& h\left(S^{2} \times D^{4}\right) \cap h^{\prime}\left(S^{2} \times D^{4}\right)=h\left(S^{2} \times S^{3}\right)=h^{\prime}\left(S^{2} \times S^{3}\right) .
\end{aligned}
$$

Proof. Let $A=h\left(S^{2} \times D^{4}\right)$ and $U=M-A$. Since $A$ is homeomorphic to $S^{2} \times D^{4}, H^{3}(A)=H^{4}(A)=0$. Moreover, $U$ is nonnull, connected and open. Since $M$ is simply connected and since $U$ is homeomorphic to $M-h\left(S^{2} \times\{0\}\right)$, the complement of a submanifold in $M$ of codimension 4 , it follows that $U$ is simply connected.

As seen in $[7$, p. 20-04], there is a commutative diagram

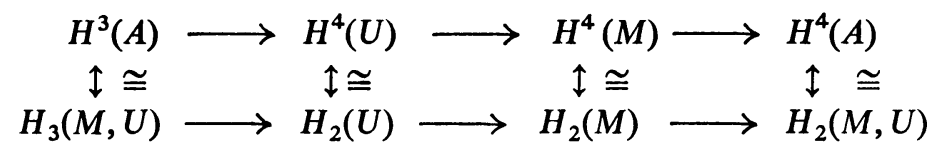

in which the upper row is the cohomology sequence of $(M, A)$, the lower row is the homology sequence of $(M, U)$ and vertical homomorphisms are the isomorphism onto in Alexander-Pontrjagin duality. Since $H^{3}(A)=H^{4}(A)=0$, it follows that the homomorphism $\mathrm{H}_{2}(U) \rightarrow \mathrm{H}_{2}(M)$ induced by the inclusion map is an isomorphism onto. Hence, by Lemma 1 , there is a primary imbedding $j^{\prime}: S^{2} \rightarrow M$ with $j^{\prime}\left(S^{2}\right) \subset U$.

It is not hard to see that the inclusion map of $j^{\prime}\left(S^{2}\right)$ into $\bar{U}$ induces an isomorphism of $H_{i}\left(j^{\prime}\left(S^{2}\right)\right)$ onto $H_{i}(\bar{U})$ for all $i$. We infer that $H_{i}\left(\bar{U}, j^{\prime}\left(S^{2}\right)\right)=0$ for all $i$. Since both $\bar{U}$ and $j^{\prime}\left(S^{2}\right)$ are connected and simply connected, it follows from the Hurewicv isomorphism theorem [4, p. 166] that $\pi_{i}\left(\bar{U}, j^{\prime}\left(S^{2}\right)\right)=0$ for all $i$. Hence $j^{\prime}\left(S^{2}\right)$ is a deformation retract of $\bar{U}$ (see, for example, [4, p. 198]). Because of this result and the fact that both $j^{\prime}\left(S^{2}\right)$ and $\bar{U}-U$ are simply connected, we may apply Smale's theorem [8] to conclude that $\bar{U}$ is diffeomorphic to a closed tubular neighborhood of $j^{\prime}\left(S^{2}\right)$, or equivalently that there is a primary imbedding $h^{\prime}: S^{2} \times D^{4} \rightarrow M$ such that $h^{\prime}\left(S^{2} \times D^{4}\right)=\bar{U}$. Hence our assertion follows.

\section{Two characterizations of $\mathrm{HCP}^{3}$ 's.}

LEMMA 6. Given any free differentiable action of the circle group $G$ on a homotopy 7-sphere $X$, the orbit space $X / G$ is an $H C P^{3}$. Conversely, given any $M$ which is an $\mathrm{HCP}^{3}$, there is a free differentiable action of the circle group on a homotopy 7-sphere such that the orbit space is diffeomorphic to $M$, and such an action is unique up to an equivariant diffeomorphism.

Proof. Suppose that a free differentiable action of the circle group $G$ on a homotopy 7 -sphere $X$ is given. Then the projection

$$
\pi: X \rightarrow X / G
$$

is a circle fibration of $X$. Therefore it is easy to see that $X / G$ is simply connected. 
Making use of Gysin's cohomology sequence [4, p. 280], we can easily verify $H^{*}(X / G)$ is isomorphic to $H^{*}\left(C P^{3}\right)$ (see (2)). Hence $X / G$ is an $\mathrm{HCP}^{3}$.

Conversely suppose that $M$ is any given $\mathrm{HCP}^{3}$. By Lemma 2, there is a primary imbedding $j: S^{2} \rightarrow M$. Let $f^{\prime}$ be an imbedding of $j\left(S^{2}\right)$ into $C P^{3}$, say $f^{\prime} j\left(S^{2}\right)$ $=$ complex projective 1-space. Since $f_{*}^{\prime}: \pi_{2}\left(j\left(S^{2}\right)\right) \rightarrow \pi_{2}\left(C P^{3}\right)$ is an isomorphism onto and since $\pi_{i}\left(C P^{3}\right)=0$ for $i=3,4,5,6$, we can make use of obstruction theory to construct an extension $f: M \rightarrow C P^{3}$ of $f^{\prime}$. Clearly $f$ may be replaced by a differentiable approximation so that we assume $f$ differentiable. Therefore $f$ induces a differentiable principal bundle over $M$ of fibre $G$, say $\pi: X \rightarrow M$. The bundle structure determines a free differentiable action of the circle group $G$ on $X$ such that the orbit space $X / G$ is diffeomorphic to $M$. Since $f^{*}: H^{*}\left(C P^{3}\right) \rightarrow H^{*}(M)$ is an isomorphism onto, if follows from Gysin's sequence that $f$ induces an isomorphism of $H^{*}\left(S^{7}\right)$ onto $H^{*}(X)$. Hence our assertion is proved.

LEMMA 7. Whenever $M$ is an $H C P^{3}$ and $h, h^{\prime}: S^{2} \times D^{4} \rightarrow M$ are primary imbeddings such that

$$
\begin{aligned}
& h\left(S^{2} \times D^{4}\right) \cup h^{\prime}\left(S^{2} \times D^{4}\right)=M, \\
& h\left(S^{2} \times D^{4}\right) \cap h^{\prime}\left(S^{2} \times D^{4}\right)=h\left(S^{2} \times S^{3}\right)=h^{\prime}\left(S^{2} \times S^{3}\right),
\end{aligned}
$$

the map $f: S^{2} \times S^{3} \rightarrow S^{2} \times S^{3}$ defined by $f=h^{\prime-1} h$ is a diffeophism satisfying (7).

Proof. By Lemma 6, we may regard $M$ as the orbit space of a free differentiable action of the circle group $G$ on a homotopy 7 -sphere $X$. Let $\pi: X \rightarrow M$ be the projection. We first claim that for any primary imbedding $j: S^{2} \rightarrow M, \pi^{-1} j\left(S^{2}\right)$ is diffeomorphic to $S^{3}$.

It is well known that $\pi: X \rightarrow M$ may be regarded as the induced bundle of a differentiable map $f: M \rightarrow C P^{3}$ (because $\pi: S^{7} \rightarrow C P^{3}$ is a universal circle bundle for manifolds of dimension $\leqq 6$ ). Since $X$ is a homotopy 7-sphere, it follows from Gysin's sequence that $f_{*}: H_{2}(M) \rightarrow H_{2}\left(C P^{3}\right)$ is an isomorphism onto. Therefore $f \mid j\left(S^{2}\right)$ induces an isomorphism of $\pi_{2}\left(j\left(S^{2}\right)\right)$ onto $\pi_{2}\left(C P^{3}\right)$ so that $f \mid j\left(S^{2}\right)$ is homotopic to a map which maps $j\left(S^{2}\right)$ homeomorphically onto the complex projective 1-space. Hence $\pi^{-1} j\left(S^{2}\right)$ is a 3-sphere.

Let $h: S^{2} \times D^{4} \rightarrow M$ be a primary imbedding. Then for any $y \in D^{4}, x \rightarrow h(x, y)$ is a primary imbedding of $S^{2}$ into $M$ so that $\pi^{-1} h\left(S^{2} \times\{y\}\right)$ is diffeomorphic to $S^{3}$. Let $Y=\pi^{-1} h\left(S^{2} \times D^{4}\right)$. Then $h^{-1}$ maps $\pi(Y)$ diffeomorphically onto $S^{2} \times D^{4}$. Let $p: S^{2} \times D^{4} \rightarrow S^{2}$ and $p^{\prime}: S^{2} \times D^{4} \rightarrow D^{4}$ be projections and $\pi_{1}: S^{3} \rightarrow S^{2}$ the natural projection (see (1)). Then $p h^{-1} \pi$ is a differentiable map of $Y$ into $S^{2}$ which induces a trivial homomorphism of $\mathrm{H}_{2}(Y)$ into $\mathrm{H}_{2}\left(S^{2}\right)$. It follows from [4, Lemma 6.4] that there is a differentiable map $k_{1}: Y \rightarrow S^{3}$ such that $\pi_{1} k_{1}=p h^{-1} \pi$. Let $k_{2}: Y \rightarrow D^{4}$ be defined by $k_{2}=p^{\prime} h^{-1} \pi$. Then $k: Y \rightarrow S^{3} \times D^{4}$ defined by $k(u)$ $=\left(k_{1}(u), k_{2}(u)\right)$, is a bundle map corresponding to $h^{-1}: \pi(Y) \rightarrow S^{2} \times D^{4}$, where the fibration $\pi: S^{3} \times D^{4} \rightarrow S^{2} \times D^{4}$ is given by $\pi(x, y)=\left(\pi_{1} x, y\right)$. Since $h^{-1}$ is a 
diffeomorphism of $\pi(Y)$ onto $S^{2} \times D^{4}, k$ is a diffeomorphism of $Y$ onto $S^{3} \times D^{4}$.

Let $Y^{\prime}=\pi^{-1} h^{\prime}\left(S^{2} \times D^{4}\right)$. Similarly, we have a diffeomorphism $k^{\prime}: Y^{\prime} \rightarrow S^{3} \times D^{4}$ which is a bundle map corresponding to $h^{\prime}: \pi\left(Y^{\prime}\right) \rightarrow S^{2} \times D^{4}$. Let $\alpha$ and $\beta$ be the elements of $H_{3}\left(S^{3} \times S^{3}\right)$ represented by $S^{3} \times\{y\}$ and $\{x\} \times S^{3}$ respectively, where $x$ and $y$ are arbitrary elements of $S^{3}$. Let $f^{f}: S^{3} \times S^{3} \rightarrow S^{3} \times S^{3}$ be defined by $f^{\prime}=k^{\prime} k^{-1}$ and $f_{*}^{*}: H_{3}\left(S^{3} \times S^{3}\right) \rightarrow H_{3}\left(S^{3} \times S^{3}\right)$ the induced homomorphism. Since $h$ and $h^{\prime}$ are strongly diffeotopic in $M, f_{*}^{\prime}(\alpha)=\alpha$. Since $X=Y \cup Y^{\prime}$ is a homotopy 7 -sphere, we can use the Mayer-Vietoris sequence of $\left(X ; Y, Y^{\prime}\right)$ to show that $\tilde{f}_{*}(\beta)=\alpha-\beta$. Hence $f: S^{2} \times S^{3} \rightarrow S^{2} \times S^{3}$ defined by $f=h^{\prime-1} h$ is a diffeomorphism satisfying (7).

Lemma 8. Giving any diffeomorphism $f: S^{2} \times S^{3} \rightarrow S^{2} \times S^{3}$ satisfying (7),

$$
M=\left(S^{2} \times D^{4}\right) \cup \cup_{f}\left(S^{2} \times D^{4}\right)
$$

is an $H C P^{3}$.

Proof. Let $\pi: S^{3} \times S^{3} \rightarrow S^{2} \times S^{3}$ be the projection defined by $\pi(x, y)$ $=\left(\pi_{1} x, y\right)$, where $\pi_{1}$ is the natural projection (see (1)). As in the proof of Lemma 7 , there is a diffeomorphism $f: S^{3} \times S^{3} \rightarrow S^{3} \times S^{3}$ which is a bundle map corresponding to $f$. Let $X=\left(S^{3} \times D^{4}\right) \cup_{f}\left(S^{3} \times D^{4}\right)$. It is easily seen that $X$ is simply connected and that there is a natural circle fibration $\pi: X \rightarrow M$.

As before, we denote by $\alpha$ and $\beta$ the element of $H_{3}\left(S^{3} \times S^{3}\right)$ represented by $S^{3} \times\{y\}$ and $\{x\} \times S^{3}$ respectively. We infer from (7) that $f_{*}(\alpha)=\alpha$ and $f_{*}(\beta)$ $=\alpha-\beta$. Making use of Mayer-Vietoris sequence, we have $H_{*}(X) \cong H_{*}\left(S^{7}\right)$. Hence $X$ is a homotopy 7 -sphere and consequently, by Lemma $6, M$ is an $\mathrm{HCP}^{3}$.

7. The group structure on $\Pi$.

Lemma 9. Let $M$ and $N$ be $H C P^{3}$ 's, $h: S^{2} \times D^{4} \rightarrow M$ and $k: S^{2} \times D^{4} \rightarrow N$ primary imbeddings, $f: S^{2} \times S^{3} \rightarrow S^{2} \times S^{3}$ the diffeomorphism of (4) and $\lambda: h\left(S^{2} \times S^{3}\right) \rightarrow k\left(S^{2} \times S^{3}\right)$ the diffeomorphism defined by $\lambda=k f h^{-1}$. Then

$$
M * N=\left(M-h\left(S^{2} \times E^{4}\right)\right) \cup_{\lambda}\left(N-k\left(S^{2} \times E^{4}\right)\right)
$$

is an $H C P^{3}$ which, up to a diffeomorphism, is independent of the choice of $h$ and $k$ so that it is uniquely determined by $M$ and $N$.

The manifold $M * N$ is called the sum of $M$ and $N$ which is different from the ordinary connected sum $M \# N$.

Proof. By Lemma 5, there are primary imbeddings $h^{\prime}: S^{2} \times D^{4} \rightarrow M$ and $k^{\prime}: S^{2} \times D^{4} \rightarrow N$ such that $h^{\prime}\left(S^{2} \times D^{4}\right)=M-h\left(S^{2} \times E^{4}\right)$ and $k^{\prime}\left(S^{2} \times D^{4}\right)$ $=N-k\left(S^{2} \times E^{4}\right)$. Applying Lemma 7, we know that $h^{-1} h^{\prime}$ and $k^{\prime-1} k$ satisfy (7). It follows that $k^{\prime-1}\left(k f h^{-1}\right) h^{\prime}=\left(k^{\prime-1} k\right) f\left(h^{-1} h^{\prime}\right)$ satisfies (7). Hence, by Lemma $8, M * N$ is an $\mathrm{HCP}^{3}$. 
Since any two primary imbeddings of $S^{2} \times D^{4}$ into an $\mathrm{HCP}^{3}$ are strongly diffeotopic (Lemma 4), that $M * N$ is uniquely determined by $M$ and $N$ can be proved by the same argument used for the ordinary connected sum $M \# N$.

LEMMA 10. The set $\Pi$ of all diffeomorphism classes of $\mathrm{HCP}^{3}$ 's can be made an abelian group under the binary operation $[M]+[N]=[M * N]$.

Proof. We recall that whenever $M$ is an $\mathrm{HCP}^{3},[M]$ denotes the diffeomorphism class of $M$. By Lemma 9 , the binary operation in $\Pi$ is well denfied. It is a direct consequence of the definition that the binary operation is associative and commutative.

Next we show that $\Pi$ has a zero. Let $h, h^{\prime}: S^{2} \times D^{4} \rightarrow C P^{3}$ be the primary imbeddings of (3), $N$ an arbitrary $\mathrm{HCP}^{3}$ and $k: S^{2} \times D^{4} \rightarrow N$ a primary imbedding. It is easily seen that there is a diffeomorphism $\zeta$ of

$$
C P^{3} * N=\left(C P^{3}-h\left(S^{2} \times E^{4}\right)\right) \cup\left(N-k\left(S^{2} \times E^{4}\right)\right)
$$

onto $N$ defined by

$$
\zeta(u)= \begin{cases}k h^{\prime-1}(u) & \text { if } u \in C P^{3}-h\left(S^{2} \times E^{4}\right), \\ u & \text { otherwise. }\end{cases}
$$

Hence $\left[C P^{3}\right]$ is the zero of $\Pi$.

To show that each $[M] \in \Pi$ has a negative, we let $h, h^{\prime}: S^{2} \times D^{4} \rightarrow M$ be primary imbeddings such that $h^{\prime}\left(S^{2} \times D^{4}\right)=M-h\left(S^{2} \times E^{4}\right)$ and $f$ the diffeomorphism of (4). Then $f^{\prime}: S^{2} \times S^{3} \rightarrow S^{2} \times S^{3}$ defined by $f^{\prime}=f h^{\prime-1} h f^{-1}$, satisfies (7) so that, by Lemma $8, N=\left(S^{2} \times D^{4}\right) \cup f^{\prime}\left(S^{2} \times D^{4}\right)$ is an $\mathrm{HCP}^{3}$. If $k: S^{2} \times D^{4} \rightarrow N$ is the primary imbedding mapping $S^{2} \times D^{4}$ identically onto the first copy of $S^{2} \times D^{4}$ in $N$ and $\lambda: h\left(S^{2} \times S^{3}\right) \rightarrow S^{2} \times S^{3}$ is defined by $\lambda=f^{\prime} f h^{-1}$, then

$$
M * N=h^{\prime}\left(S^{2} \times D^{4}\right) \cup_{\lambda}\left(S^{2} \times D^{4}\right) .
$$

Since $f^{\prime} f h^{-1}=f h^{-1}$ and since, by (9), $\left(S^{2} \times D^{4}\right) \cup_{f}\left(S^{2} \times D^{4}\right)$ is diffeomorphic to $C P^{3}$, it follows that $M * N$ is diffeomorphic to $C P^{3}$. This proves that $[N]$ is the negative of $[M]$.

8. A correspondence between HCP $^{3}$ 's and $(6,3)$-sphere pairs. We recall that $\Sigma$ denotes the set of all diffeomorphism classes of $(6,3)$-sphere pairs and that the diffeomorphism class of each $(6,3)$-sphere pair $(P, Q)$ is denoted by $[P, Q]$. As seen in [3], for any two $(6,3)$-sphere pairs $\left(P^{\prime}, Q^{\prime}\right)$ and $\left(P^{\prime \prime}, Q^{\prime \prime}\right)$, a connected sum $\left(P^{\prime} \# P^{\prime \prime}, Q^{\prime} \# Q^{\prime \prime}\right)$ can be constructed as follows. Consider $R^{3}$ as a subset of $R^{6}=R^{3} \times R^{3}$ by identifying each $y \in R^{3}$ with $(0, y) \in R^{6}$. Then $D^{3}$ is a submanifold of $D^{6}$ and we have a $(6,3)$-disk pair $\left(D^{6}, D^{3}\right)$. Let $h:\left(D^{6}, D^{3}\right) \rightarrow\left(P^{\prime}, Q^{\prime}\right)$ and $k:\left(D^{6}, D^{3}\right) \rightarrow\left(P^{\prime \prime}, Q^{\prime \prime}\right)$ be orientation-preserving imbeddings, $f:\left(D^{6}, D^{3}\right) \rightarrow\left(D^{6}, D^{3}\right)$ the reflection with respect to a hyperplane through the origin and 


$$
\lambda:\left(h\left(S^{5}\right), h\left(S^{2}\right)\right) \rightarrow\left(k\left(S^{5}\right), k\left(S^{2}\right)\right)
$$

the diffeomorphism defined by $\tau=k f h^{-1}$. Then we have a $(6,3)$-sphere pair $(P, Q)$ defined by

$$
\begin{aligned}
& P=\left(P^{\prime}-h\left(E^{6}\right)\right) \cup_{\tau}\left(P^{\prime \prime}-k\left(E^{6}\right)\right), \\
& Q=\left(P^{\prime \prime}-h\left(E^{3}\right)\right) \cup_{\tau}\left(Q^{\prime \prime}-k\left(E^{3}\right)\right) .
\end{aligned}
$$

The $(6,3)$-sphere pair $(P, Q)$, up to a diffeomorphism is uniquely determined by $\left(P^{\prime}, Q^{\prime}\right)$ and $\left(P^{\prime \prime}, Q^{\prime \prime}\right)$ and is defined to be $\left(P^{\prime} \# P^{\prime \prime}, Q^{\prime} \# Q^{\prime \prime}\right)$. It is easy to verify that $\Sigma$ can be made an abelian group under the binary operation

$$
\left[P^{\prime}, Q^{\prime}\right]+\left[P^{\prime \prime}, Q^{\prime \prime}\right]=\left[P^{\prime} \# P^{\prime \prime}, Q^{\prime} \# Q^{\prime \prime}\right] \text {. }
$$

As proved in [3], there is a homomorphism of $\Sigma$ onto $Z$. Hence $\Sigma$ is an abelian group containing an element of infinite order.

LEMMA 11. If $M$ is an $H C P^{3}, h: S^{2} \times D^{4} \rightarrow M$ is a primary imbedding, $f$ is the diffeomorphism of (4) and $\alpha: h\left(S^{2} \times S^{3}\right) \rightarrow S^{2} \times S^{3}$ is the diffeomorphism defined by $\alpha=f h^{-1}$, then we have a $(6,3)$-sphere pair $(P, Q)$ in which

$$
\begin{aligned}
& P=\left(M-h\left(S^{2} \times E^{4}\right)\right) \cup_{\alpha}\left(D^{3} \times S^{3}\right), \\
& \left.Q=\{0\} \times S^{3} \quad \text { (as a subset of } D^{3} \times S^{3}\right) .
\end{aligned}
$$

Moreover, the diffeomorphism class $[P, Q]$ is uniquely determined by the diffeomorphism class $[M]$. Furthermore, the map $[M] \rightarrow[P, Q]$ is a homomorphism

$$
\phi: \Pi \rightarrow \Sigma .
$$

Proof. The Mayer-Vietoris sequence of $\left(P ; M-h\left(S^{2} \times E^{4}\right), D^{3} \times S^{3}\right)$ shows that $H^{*}(P)$ is isomorphic to $H^{*}\left(S^{6}\right)$. Since $P$ is simply connected, it is a homotopy 6 -sphere so that it is diffeomorphic to $S^{6}[9]$. Hence $(P, Q)$ is a $(6,3)$-sphere pair. That $[P, Q]$ is uniquely determined by $[M]$ is a consequence of Lemma 4 . Hence it remains to be shown that $\phi$ is a homomorphism.

Let $M$ and $N$ be $\mathrm{HCP}^{3}$ 's. Let $h: S^{2} \times D^{4} \rightarrow M$ and $k: S^{2} \times D^{4} \rightarrow N$ be primary imbeddings, $f$ the diffeomorphism of (4),

$$
\alpha: h\left(S^{2} \times S^{3}\right) \rightarrow S^{2} \times S^{3} \text { and } \beta: k\left(S^{2} \times S^{3}\right) \rightarrow S^{2} \times S^{3}
$$

defined by $\alpha=f h^{-1}$ and $\beta=f k^{-1}$ and

$$
\begin{array}{ll}
P^{\prime}=\left(M-h\left(S^{2} \times E^{4}\right)\right) \cup_{\alpha}\left(D^{3} \times S^{3}\right), & Q^{\prime}=\{0\} \times S^{3} ; \\
P^{\prime \prime}=\left(N-k\left(S^{2} \times E^{4}\right)\right) \cup_{\beta}\left(D^{3} \times S^{3}\right), & Q^{\prime \prime}=\{0\} \times S^{3} .
\end{array}
$$

Then $\phi[M]=\left[P^{\prime}, Q^{\prime}\right]$ and $\phi[N]=\left[P^{\prime \prime}, Q^{\prime \prime}\right]$. 
By Lemma 5, there is a primary imbedding $h^{\prime}: S^{2} \times D^{4} \rightarrow M$ with

$$
h^{\prime}\left(S^{2} \times D^{4}\right)=M-h\left(S^{2} \times E^{4}\right) \text {. }
$$

It is clear that $P^{\prime}$ is diffeomorphic to $\left(S^{2} \times D^{4}\right) \cup_{\alpha^{\prime}}\left(D^{3} \times S^{3}\right)$ so that we may set

$$
P^{\prime}=\left(S^{2} \times D^{4}\right) \cup_{\alpha^{\prime}}\left(D^{3} \times S^{3}\right),
$$

where $\alpha^{\prime}=\alpha h^{\prime}=f\left(h^{-1} h^{\prime}\right)$. Let $p, p^{\prime}$ and $q$ be as in (5) and (6). By Lemma $7, f$ and $h^{-1} h^{\prime}$ satisfy (7). We infer that $p \alpha^{\prime} q: S^{3} \rightarrow S^{2}$ is homotopic to a constant map and $p^{\prime} \alpha^{\prime} q: S^{3} \rightarrow S^{3}$ is of degree 1 . Let

$$
\begin{aligned}
& H_{+}^{4}=\left\{\left(x_{1}, x_{2}, x_{3}, x^{4}\right) \in D_{4} \mid x_{4} \geqq 0\right\}, \\
& D_{+}^{3}=S^{3} \cap H_{+}^{4} .
\end{aligned}
$$

Then $\alpha^{\prime}: S^{2} \times S^{3} \rightarrow S^{2} \times S^{3}$ is diffeotopic to a diffeomorphism which is identity on $S^{2} \times D_{+}^{3}$ so that we may set

$$
\alpha^{\prime} \mid\left(S^{2} \times D_{+}^{3}\right)=\text { identity. }
$$

Similarly, there is a primary imbedding $k^{\prime}: S^{2} \times D^{4} \rightarrow N$ with $k^{\prime}\left(S^{2} \times D^{4}\right)$ $=N-k\left(S^{2} \times E^{4}\right)$ so that we may set

$$
P^{\prime \prime}=\left(S^{2} \times D^{4}\right) \cup_{\beta^{\prime}}\left(D^{3} \times S^{3}\right)
$$

with $\beta^{\prime}=\beta k^{\prime}=f k^{-1} k^{\prime}$. Moreover, if we let

$$
H_{-}^{4}=\operatorname{cl}\left(D^{4}-H_{+}^{4}\right), \quad D_{-}^{3}=S^{3} \cap H_{-}^{4},
$$

we may assume that

$$
\beta^{\prime} \mid\left(S^{2} \times D_{-}^{3}\right)=\text { identity. }
$$

By definition, we may let

$$
M * N=\left(M-h\left(S^{2} \times E^{4}\right)\right) \cup_{\lambda}\left(N-k^{\prime}\left(S^{2} \times E^{4}\right)\right)
$$

with $\lambda=k^{\prime} f h^{-1}$. Therefore, if $\phi[M * N]=[P, Q]$, we may let

$$
P=\left((M * N)-k\left(S^{2} \times E^{4}\right)\right) \cup_{\gamma}\left(D^{3} \times S^{3}\right)
$$

or

$$
P=\left(S^{2} \times D^{4}\right) \cup \gamma^{\prime}\left(D^{3} \times S^{3}\right),
$$

where $\gamma=f k^{-1}$ and $\gamma^{\prime}=f k^{-1} \lambda h^{\prime}=\left(f k^{-1} k^{\prime}\right)\left(f h^{-1} h^{\prime}\right)=\beta^{\prime} \alpha^{\prime}$.

Now we observe that in $P^{\prime},\left(S^{2} \times H_{+}^{4}\right) \cup_{\alpha^{\prime}}\left(D^{3} \times D_{+}^{3}\right)$, after the corner along $S^{2} \times\left(D_{+}^{3} \cap D_{-}^{3}\right)$ being straightened, is diffeomorphic to $D^{6}$. In fact, we have a diffeomorphism

$$
\xi: D^{6} \rightarrow\left(S^{2} \times H_{+}^{4}\right) \cup_{\alpha^{\prime}}\left(D^{3} \times D_{+}^{3}\right)
$$


such that for any $(x, y) \in\left(S^{2} \times H_{+}^{4}\right) \cup_{\alpha^{\prime}}\left(D^{3} \times D_{+}^{3}\right)$, if $x /\left(|x|^{2}+|y|^{2}\right)^{1 / 2}$ $=\left(x_{1}^{\prime}, x_{2}^{\prime}, x_{3}^{\prime}\right)$ and $y /\left(|x|^{2}+|y|^{2}\right)^{1 / 2}=\left(y_{1}^{\prime}, y_{2}^{\prime}, y_{3}^{\prime}, y_{4}^{\prime}\right)$, then

$$
\xi^{-1}(x, y)=\left(x_{1}^{\prime}, x_{2}^{\prime}, x_{3}^{\prime}, y_{1}^{\prime}, y_{2}^{\prime}, y_{3}^{\prime}\right) \text {. }
$$

The diffeomorphism $\xi$ is also regarded as an imbedding of $D^{6}$ into $P^{\prime}$. Similarly, we have a diffeomorphism

$$
\eta: D^{6} \rightarrow\left(S^{2} \times H_{-}^{4}\right) \cup_{\beta^{\prime}}\left(D^{3} \times D_{-}^{3}\right)
$$

which is defined just like $\xi$ and is regarded as an imbedding of $D^{6}$ into $P^{\prime \prime}$. Since exactly one of $\xi$ and $\eta$, say $\xi$, is orientation-preserving, we may let

$$
P^{\prime} \# P^{\prime \prime}=\left(P^{\prime}-\xi\left(E^{6}\right)\right) \cup_{\tau}\left(P^{\prime \prime}-\eta\left(E^{6}\right)\right)
$$

with $\tau=\eta \xi^{-1}$. By (14) and (16),

$$
\begin{aligned}
& P^{\prime}-\xi\left(E^{6}\right)=\left(S^{2} \times H_{-}^{4}\right) \cup_{\alpha^{\prime}}\left(D^{3} \times D_{-}^{3}\right), \\
& P^{\prime \prime}-\eta\left(E^{6}\right)=\left(S^{2} \times H_{+}^{4}\right) \cup_{\beta^{\prime}}\left(D^{3} \times D_{+}^{3}\right) .
\end{aligned}
$$

It follows from (15), (17) and (18) that

$$
P^{\prime} \# P^{\prime \prime}=\left(S^{2} \times D^{4}\right) \cup_{\beta^{\prime} \alpha^{\prime}}\left(D^{3} \times S^{3}\right)=P .
$$

That $Q^{\prime} \# Q^{\prime \prime}=Q$ is obvious from our construction. Hence the proof is completed.

LEMMA 12. If $(P, Q)$ is a $(6,3)$-sphere pair, $p, p^{\prime}$ and $q$ are maps of (5) and (6), then there are orientation-preserving imbeddings

$$
k: S^{2} \times D^{4} \rightarrow P, \quad k^{\prime}: D^{3} \times S^{3} \rightarrow P
$$

such that (i) $p\left(k^{\prime-1} k\right) q: S^{3} \rightarrow S^{2}$ is homotopic to a constant map and $p^{\prime}\left(k^{\prime-1} k\right) q$ : $S^{3} \rightarrow S^{3}$ is of degree -1 and (ii) $k^{\prime}\left(\{0\} \times S^{3}\right)=Q$. Moreover, if $k$ is as above, $f$ is the diffeomorphism of (4) and $\lambda: k\left(S^{2} \times S^{3}\right) \rightarrow S^{2} \times S^{3}$ is defined by $\lambda=f k^{\prime-1}$, then

$$
M=k\left(S^{2} \times D^{4}\right) \cup_{\lambda}\left(S^{2} \times D^{4}\right)
$$

is an $H C P^{3}$. Furthermore, $\phi[M]=[P, Q]$.

Proof. Since any vector bundle over a 3 -sphere is a product bundle [6, p. 137] there is an orientation-preserving imbedding $k^{\prime}: D^{3} \times S^{3} \rightarrow P$ which defines an orientation-preserving diffeomorphism of $\{0\} \times S^{3}$ onto $Q$.

Let $j: S^{2} \rightarrow P$ be an imbedding with $j\left(S^{2}\right)=P-k^{\prime}\left(D^{3} \times S^{3}\right)$ and such that $j\left(S^{2}\right)$ has linking number 1 with $Q$. It is easy to see that $H_{i}\left(P-k^{\prime}\left(E^{3} \times S^{3}\right), j\left(S^{2}\right)\right)$ $=0$ for all $i$. Therefore $j\left(S^{2}\right)$ is a deformation retract of $P-k^{\prime}\left(E^{3} \times S^{3}\right)$. By Smale's theorem [8], there is an orientation-preserving imbedding $k: S^{2} \times D^{4} \rightarrow P$ with $k\left(S^{2} \times D^{4}\right)=P-k^{\prime}\left(E^{3} \times S^{3}\right)$ and such that (i) $k(x, 0)=j(x)$ and (ii) the orientation of $k\left(S^{2} \times\{0\}\right)$ may be so chosen that the map of $S^{2}$ into 
$k\left(S^{2} \times\{0\}\right)$ defined by $x \rightarrow k(x, 0)$ is orientation-preserving and $k\left(S^{2} \times\{0\}\right)$ has linking number 1 with $Q$.

By our choice of $k$ and $k^{\prime}$ above, it is easily seen that $p^{\prime}\left(k^{\prime-1} k\right) q: S^{3} \rightarrow S^{3}$ is of degree -1 . However, $p\left(k^{\prime-1} k\right) q: S^{3} \rightarrow S^{2}$ may present a nonzero element $\sigma$ of $\pi_{3}\left(S^{2}\right)$. In order to have our assertion, we replace $k^{\prime}$ by an imbedding $k_{1}^{\prime}: D^{3} \times S^{3} \rightarrow P$ constructed as follows: Let $\xi: S^{3} \rightarrow \operatorname{SO}(3)$ be a differentiable map such that the composite map

$$
S^{3} \stackrel{\xi}{\rightarrow} \mathrm{SO}(3) \rightarrow \mathrm{SO}(3) / \mathrm{SO}(2)=S^{2}
$$

represents $-\sigma$ in $\pi_{3}\left(S^{2}\right)$. Then for any $(x, y) \in S^{2} \times S^{3}$, we let

$$
k_{1}^{\prime}(x, y)=k^{\prime}(\xi(y) x, y) .
$$

Clearly $p\left(k_{1}^{\prime-1} k\right) q: S^{3} \rightarrow S^{2}$ is homotopic to a constant map.

The rest of L.emma 12 follows from Lemmas 8 and 11.

L.EMMA 13. $\phi: \Pi \rightarrow \Sigma$ is onto.

Proof. It is a direct consequence of Lemmas 11 and 12.

As we have mentioned in $\S 3, \Sigma$ is an abelian group containing an element of infinite order. Hence Theorem 1 follows from Lemmas 6 and 13.

9. Actions having a fixed point set diffeomorphic to $S^{3}$. Let $X$ be a homotopy 7-sphere and let the circle group $G$ act differentiably on $X$ such that the action has a fixed point set $F$ diffeomorphic to $S^{3}$ and is free otherwise. Let $P$ be the orbit space $X / G$ and $Q=F$, regarded as a submanifold of $P$. It is not hard to see that $(P, Q)$ is a $(6,3)$-sphere pair.

Conversely suppose that a $(6,3)$-sphere pair $(P, Q)$ is given, and let

$$
k: S^{2} \times D^{4} \rightarrow P, \quad k^{\prime}: D^{3} \times S^{3} \rightarrow P
$$

be the imbeddings of Lemma 12. As seen in $\$ 2$, there is a differentiable action of the circle group $G$ on $D^{4} \times S^{3}$ which leaves every point of $\{0\} \times S^{3}$ fixed and acts freely otherwise. The projection

$$
\pi: D^{4} \times S^{3} \rightarrow D^{3} \times S^{3}
$$

induces a differentiable principal bundle

$$
\tau: S^{3} \times S^{3} \rightarrow k\left(S^{2} \times S^{3}\right)
$$

of fibre $G$ defined by $\tau=k \pi$. As before, we may assume that $\tau$ is induced by a differentiable map $f: k\left(S^{2} \times S^{3}\right) \rightarrow C P^{3}$. Since $\pi_{i}\left(C P^{3}\right)=0$ for $i=3,4,5,6$, we can use obstruction theory to construct a differentiable extension

$$
f^{\prime}: k\left(S^{2} \times D^{4}\right) \rightarrow C P^{3}
$$

of $f$. The map $f^{\prime}$ induces a differentiable principal bundle 


$$
\tau^{\prime}: Y \rightarrow k\left(S^{2} \times D^{4}\right)
$$

of fibre $G$. Since $Y$ and $D^{4} \times S^{3}$ intersect at their common boundary, it follows that

$$
X=Y \cup\left(D^{4} \times S^{3}\right)
$$

is a differentiable 7-manifold. It is not hard to show that $X$ is simply connected and has the integral cohomology group of $S^{7}$. Therefore $X$ is a homotopy 7-sphere. Clearly there is a natural differentiable action of $G$ on $X$ which has $\{0\} \times S^{3}$ as the fixed point set and is free otherwise. Moreover, $\left(X / G,\{0\} \times S^{3}\right)$ is diffeomorphic to $(P, Q)$. Hence we have proved

LEMMA 14. If the circle group $G$ acts differentiably on a homotopy 7-sphere $X$ such that the action has a fixed point set $F$ diffeomorphic to $S^{3}$ and is free otherwise, then $(X / G, F)$ is a $(6,3)$-sphere pair. Conversely, for any $(6,3)$-sphere pair $(P, Q)$, we have such an action with $(X / G, F)$ diffeomorphic to $(P, Q)$.

Denote by $(G, X, F)$ a differentiable action of the circle group $G$ on a homotopy 7 -sphere $X$ which has a fixed point set $F$ diffeomorphic to $S^{3}$ and is free otherwise. The equivariant diffeomorphic class of $(G, X, F)$ is denoted by $[G, X, F]$. Let $\Sigma^{*}$ be the totality of $[G, X, F]$ 's. It is clear that there is a function

$$
\psi: \Sigma^{*} \rightarrow \Sigma
$$

defined by $\psi[G, X, F]=[X / G, F]$.

Given any $\left(G, X^{\prime}, F^{\prime}\right)$ and $\left(G, X^{\prime \prime}, F^{\prime \prime}\right)$, we have given the connected sum

$$
\left(\left(X^{\prime} / G\right) \#\left(X^{\prime \prime} / G\right), F^{\prime} \# F^{\prime \prime}\right)
$$

(see $§ 8)$. It is not hard to see that a similar construction of

$$
\left(G, X^{\prime} \# X^{\prime \prime}, F^{\prime} \# F^{\prime \prime}\right)
$$

can be given by using equivariant imbeddings

$$
h:\left(D^{7}, D^{3}\right) \rightarrow\left(X^{\prime}, F^{\prime}\right) \text { and } k:\left(D^{7}, D^{3}\right) \rightarrow\left(X^{\prime \prime}, F^{\prime \prime}\right),
$$

where $G$ acts on $D^{7}$ as in the second standard action of $G$ given in $\$ 2$. Moreover,

$$
\left(X^{\prime} \not \# X^{\prime \prime}\right) / G=\left(X^{\prime} / G\right) \not=\left(X^{\prime \prime} / G\right) .
$$

LEMma 15. The set $\Sigma^{*}$ of all equivariant diffeomorphism classes $[G, X, F]$ can be made an abelian group under the binary operation

$$
\left[G, X^{\prime}, F^{\prime}\right]+\left[G, X^{\prime \prime}, F^{\prime \prime}\right]=\left[G, X^{\prime} \# X^{\prime \prime}, F^{\prime} \# F^{\prime \prime}\right] \text {. }
$$

Moreover, the function $\psi: \Sigma^{*} \rightarrow \Sigma$, defined by $\psi[G, X, F]=[X / G, F]$, is a homomorphism onto.

Proof. The first part can be proved by the same argument used for $\Sigma$. That $\psi$ 
is a homomorphism is a direct consequence of the group structures in $\Sigma^{*}$ and $\Sigma$. That $\psi$ is onto is a consequence of Lemma 14 .

Proof of Theorem 3. Let $\theta_{7}$ be the group of all diffeomorphism classes of homotopy 7 -spheres. Then we have a homomorphism

$$
\sigma: \Sigma^{*} \rightarrow \theta_{7}
$$

mapping each $[G, X, F]$ into the diffeomorphism class of $X$.

The homomorphism $\sigma$ is onto. In fact, a generator of $\theta_{7}$ is represented by the homotopy 7 -sphere $X$, given by

$$
X=\left(S^{3} \times D^{4}\right) \cup_{\lambda}\left(S^{3} \times D^{4}\right),
$$

where $\lambda: S^{3} \times S^{3} \rightarrow S^{3} \times S^{3}$ is defined by

$$
\lambda(u, v)=\left(v^{2} u v^{-1}, v\right)
$$

[10]. Let $G$ act on $X$ such that for any $g \in G$ and any $(u, v) \in X$,

$$
g(u, v)=\left(g u g^{-1}, g v g^{-1}\right) .
$$

Then the fixed point set $F$ is diffeomorphic to $S^{\mathbf{3}}$ and $G$ acts freely on $X-F$. Since $X$ represents a generator of $\theta_{7}$, our claim follows.

Since $\Sigma$ is infinite and $\psi: \Sigma^{*} \rightarrow \Sigma$ is onto, it follows that $\Sigma^{*}$ is infinite. It is known that $\theta_{7}$ is a cyclic group of order 28 (see [10] or [9]). Therefore, by the fact that $\sigma: \Sigma^{*} \rightarrow \theta_{7}$ is onto, $\sigma^{-1}[X]$ is infinite for all $[X] \in \theta_{7}$. Hence our assertion follows. Notice that the kernel of $\sigma$ actually contains an element of infinite order.

COROLLARY. There are infinitely many differentiably distinct differentiable actions of the circle group on $S^{7}$ having $S^{3}$ as the fixed point set and acting freely otherwise. Moreover, the equivariant diffeomorphism classes of these actions can be made an abelian group containing an element of infinite order.

10. Conclusion. Let $(G, X, F)$ be as in $\S 9$. Then $X$ bounds an $S$-parallelizable compact differentiable 8-manifold $K$ (see [10] or [9]). By the collaring theorem, there is a neighborhood $U$ of $X$ in $K$ homeomorphic to $X \times[0,1)$. Since $\pi_{3}(U)=0$, $F$ is contractible in $U$. It follows from $\operatorname{dim} U=2(1+\operatorname{dim} F)$ that there is an imbedding $h: D^{4} \rightarrow U$ such that $h\left(S^{3}\right)=F$ and $h\left(D^{4}\right)$ intersects $X$ orthogonally at $F$ with respect to a given Reimannian metric on $K$. The construction of $h$ is the same as in Whitney's proof that every closed differentiable $n$-manifold can be imbedded into $R^{2 n}$ [11]. If we use a Riemannian metric on $K$ which, restricted to $X$, is invariant under the action of $G$, then we have a closed tubular neighborhood of $F$ in $K$ given by an imbedding $k: D^{4} \times D^{4} \rightarrow K$ which maps $D^{4} \times S^{3}$ equivariantly into $X$ and such that for any $y \in D^{4}, k(0, y)=h(y)$. Let

$$
K^{\prime}=K-k\left(E^{4} \times D^{4}\right), \quad X^{\prime}=\text { boundary of } K^{\prime} .
$$


We may regard $K^{\prime}$ as a differentiable 8-manifold of boundary $X^{\prime}$ by straightening the corner along $k\left(S^{3} \times S^{3}\right)$. Now we have a free differentiable action of $G$ on $X^{\prime}$ such that the action on $X^{\prime} \cap X$ agrees with that on $X$ and $k: S^{3} \times D^{4} \rightarrow X^{\prime}$ is equivariant, where the action on $S^{3} \times D^{4}$ is the one in the second standard action of $\$ 2$. It is easily seen that $X^{\prime}$ is a homotopy 7 -sphere and that

$$
\phi\left[X^{\prime} / G\right]=[X / G, F]
$$

where $\phi$ is the homomorphism of Lemma 12. Since the inclusion map of $K^{\prime}$ into $K$ induces an isomorphism of $H^{4}(K)$ onto $H^{4}\left(K^{\prime}\right)$, it follows that $K$ and $K^{\prime}$ are of the same index so that $X$ and $X^{\prime}$ are diffeomorphic [10]. Hence Theorem 2 follows from Theorem 3 .

COROLLARY. There are infinitely many differentiably distinct free differentiable actions of the circle group on $S^{7}$. Moreover, the equivariant diffeomorphism classes of these actions can be made an abelian group containing an element of infinite order.

Appendix. Using recent results in a paper by Haefliger, Differentiable embeddings of $S^{n}$ in $S^{n+q}$ for $q>2$, which is at present unpublished, some of our results can be strengthened. Following Haefliger, we denote by $C_{3}^{3}$ the group of isotopy classes of imbeddings of $S^{3}$ into $S^{6}$. Clearly for each imbedding $i: S^{3} \rightarrow S^{6},\left(S^{6}, i\left(S^{3}\right)\right)$ is a $(6,3)$-sphere pair. Therefore we have a map

$$
\xi: C_{3}^{3} \rightarrow \Sigma
$$

which maps the isotopy class of $i$ into $\left[S^{6}, i\left(S^{3}\right)\right]$. It is not hard to see that $\xi$ is a homomorphism onto.

There is a map

$$
\eta: \Pi \rightarrow C_{3}^{3}
$$

defined as follows. Let $M$ be an $\mathrm{HCP}^{3}$. As in Lemma 11, we have a $(6,3)$-sphere pair $(P, Q)$ with

$$
P=\left(M-h\left(S^{2} \times E^{4}\right)\right) \cup_{\alpha}\left(D^{3} \times S^{3}\right), Q=\{0\} \times S^{3} .
$$

Therefore we have an imbedding

$$
i: S^{3} \rightarrow P
$$

defined by $i(y)=(0, y) \in Q$. Since $P$ is diffeomorphic to $S^{6}$, we may regard $i$ as an imbedding of $S^{3}$ into $S^{6}$. The isotopy class of $i$ is taken to be $\eta[M]$. Following the proof of Lemma 11, we can show that $\eta$ is a homomorphism.

The homomorphism $\eta$ is an isomorphism onto. In fact, if an imbedding $i: S^{3} \rightarrow S^{6}$ is given, we can have imbeddings

$$
k: S^{2} \times D^{4} \rightarrow S^{6}, k^{\prime}: D^{3} \times S^{3} \rightarrow S^{6}
$$


satisfying the requirements of Lemma 12 and such that

$$
k^{\prime}(0, y)=i(y) \text { for all } y \in S^{3} \text {. }
$$

Moreover, $k^{\prime}$ is unique up to a strong diffeotopy. Hence we can uniquely determine an $[M] \in \Pi$ to be mapped into the isotopy class of $i$.

From what is said above and Haefliger's theorem that $C_{3}{ }^{3} \cong Z$, we can now show that $\Pi$ and $\Sigma$ are isomorphic to $Z$ and that $\phi$ is an isomorphism. The diagram

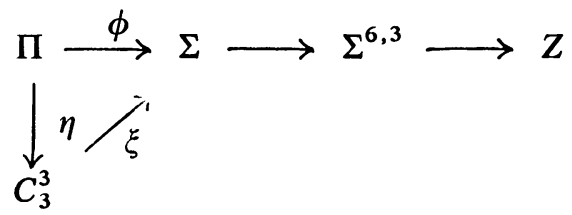

is commutative. (For the homomorphisms $\Sigma \rightarrow \Sigma^{6,3} \rightarrow Z$, see §3.) Since all homomorphisms in the diagram are onto and since $C_{3}^{3}$ is $\cong Z$, our assertion follows.

As immediate consequences of this result, the group in Theorem 1 and the groups in the two corollaries in the last two sections are isomorphic to $Z$.

If we use Cerf's result $\Gamma_{4}=0$ instead of Haefliger's result, we can show that $\phi: \Pi \rightarrow \Sigma$ is an isomorphism onto (but not that $\Pi$ and $\Sigma$ are isomorphic to $Z$ ). In fact, for any $(6,3)$-sphere pair $\left(S^{6}, Q\right)$, there is an imbedding $i: S^{3} \rightarrow S^{6}$ with $i\left(S^{3}\right)=Q$. Since $\Gamma_{4}=0, i$, as a diffeomorphism of $S^{3}$ onto $Q$, is unique up to a diffeomotopy. Therefore we may proceed as above and obtain an imbedding $k^{\prime}: D^{3} \times S^{3} \rightarrow S^{6}$ which is unique up to a strong diffeotopy.

\title{
REFERENCES
}

1. W. C. Hsiang, and W. Y. Hsiang, Some free differentiable actions of $S^{1}$ and $S^{3}$ on 11 -sphere, Quart. J. Math. 15 (1964), 371-374.

2. J. W. Milnor, Differentiable structures, Mimeographed Notes, Princeton University, Princeton, N. J., 1961.

3. A. Haefliger, Knotted ( $4 k-1)$-spheres in 6k-space, Ann. of Math. 75 (1962), 452-466.

4. S. T. Hu, Homotopy theory, Academic Press, New York, 1959.

5. A. Haefliger, Plongements differentiables de variétés dans variétés, Comment. Math. Helv. 36 (1961), 47-82.

6. N. E. Steenrod, Fibre bundles, Princeton Univ. Press, Princeton, N. J., 1951.

7. H. Cartan, Seminaire Cartan, Paris, 1950-1951.

8. S. Smale, On the structure of manifolds, Amer. J. Math. 84 (1962), 387-399.

9. M. A. Kervaire and J. W. Milnor, Groups of homotopy spheres. I, Ann. of Math. 77 (1963), 504-537.

10. J. W. Milnor, Differentiable manifolds which are homotopy spheres, Mimeographed Notes, Princeton University, Princeton, N. J., 1958.

11. H. Whitney, The self intersection of a smooth n-manifold in $2 n$-space, Ann. of Math. 45 (1944), 220-246.

\author{
INSTITUTE FOR ADVANCED STUDY, \\ PRINCETON, NeW JERSEY \\ University of Pennsylvania, \\ Philadelphia, Pennsylvania
}

